Egyptian Poultry Science Journal

http://www.epsaegypt.com

ISSN: 1110-5623 (Print) - 2090-0570 (On line)

\title{
UTILIZATION OF POTATO PEELS AS A FIBER SOURCE IN FEEDING TWO LINES OF GROWING RABBIT
}

M. A. Kairalla ${ }^{1}$; M. Bahie El-Deen ${ }^{1}$; Mona Osman, M. ${ }^{1}$; A. M. El-Raffa ${ }^{1}$; S. A. El-Safty ${ }^{2}$

${ }^{1}$ Poultry prod. Dept., Fac. of Agric., Alexandria Univ.

${ }^{2}$ Poultry prod. Dept., Fac. of Agric., Ain Shams Univ.

Corresponding author: Majdy Kairalla; E-mail: majdiabdelfaraj@yahoo.com

\section{Received: 01/04/2016 Accepted: 08/05/2016}

\begin{abstract}
Total numbers of 300 growing V. and Alex. rabbits at age of 4-weeks were used to study the effect of dietary potato peels as a partial replacement at levels 5, 10, 15 and $20 \%$ of dietary fiber inclusion. Rabbits of each line were randomly classified into five equal groups (30 each). The 1th group received the basal diet and served as control diet. The other four groups received the basal diet with replacement potato peels meal at the studied levels, respectively. The chemical composition of the potato peels meal clearly shows that it contains $14 \%$ crude protein, $15.6 \%$ crude fiber and $57.1 \%$ nitrogen free extract and these compounds falls within ranges of studied wheat brain and Alfalfa hay. While, ether extract and ash content of potato peels meal were higher than those of other studied materials. Significant difference was recorded for body weight, gain, feed intake and feed conversion as a result of lines difference over all the experimental growth period (4-10) week old. In that, average body weight of Alex. rabbits and its gain were higher than those of V. line. The opposite was true with feed intake, where rabbits of V. line consumed more diet compared with those of Alex. line. Dietary potato peels meal treatments $(10 \%)$ significantly increased body weight and weight gain and consequently feed intake was also increased. Meanwhile, the best score of feed conversion was attained with Alex. rabbits fed inclusion level of $10 \%$ potato peels meal. This trend did not continue but it significantly decreased as increasing inclusion level up to $20 \%$ and resulted to impaired feed conversion which being 3. 89 for V. rabbits and followed by 3.37 for Alex. rabbits. The relative economic efficiency of the different formulated diets as affected by dietary treatments of 5 and $10 \%$ were highly by 8.9 and $16.1 \%$ over the corresponding of the control group for Alex. rabbits and followed by 7.2 and $5.5 \%$ over the control group of V. rabbits, respectively. In conclusion, potato peel meal can be used as a good alternative source of fiber at levels of 5 and $10 \%$ in growing V. and Alex. rabbits diets, respectively with a good influence on productive performance and can be considered a cheap of fiber ingredients in rabbits diets formations.
\end{abstract}

Keywords: Potato Peels - Rabbits - Productive Performance - Economic efficiency. 


\section{INTRODUCTION}

A rabbit production has become a major sector of the poultry production as well as its improvement is considered one of the main targets of both public and private sectors. Advances in genetic, nutrition and husbandry management over the same period have resulted in a phenomenal improvement in the productivity. In this respect, chronic feed deficient represent to animal production in many of the developing countries, especially Egypt, due to population increase and consequently allocation of available for cereal production, thereby reducing the availability of cultivated land for animal fodder production. Besides that, rabbits have high ability to consume forage and agricultural by-products contained high levels of fiber (Cheeke, 1986). This situation demands the use of unconventional feed resources in livestock ration, especially from Agro-industrial byproducts. Inclusions of these materials are often severely limited because little information is available about their nutritive value (Shamma et al., 2014). Vegetable peels are usually considered waste, so they are obviously cost-effective (Parmar and Kar, 2009; Hamendra et al., 2010). Among these materials which may possess a considerable feeding value is the potato peels where, potato annual output is around 206 million tons which is the largest in Africa (FAO, 2006). In Egypt, the yield of potato crop was two million tons (A.E.S.I. 2008). Approximately $35 \%$ of total processed potato crop is discarded as a waste during processing (El-Boushy and Van der Poel, 1994). However, Smith and Huxsoll (1987) estimated the peeling losses of the potato chips industry which used abrasion peeling extensively to be $10 \%$. This material is found to be a good source of dietary fiber and polyphenols (Singh et al., 2005), which is strongly antioxidant (Singh et al., 2008). In this respect, Chlorogenic $(50.31 \%)$, gallic $(41.67 \%)$, protocatechuic $(7.82 \%)$ and caffeic acid $(0.21 \%)$ were the major phenolic compounds detected in the study conducted by Rodriguez De Sotillo et al. (1994). Moreover, the extract of potato peel had both bactericidal and bacteriostatic effects but only at a high concentration. Researches on the use of vegetable and fruits peels are on the increased. Amber et al. (2002) showed that types of dietary fiber mainly affected the digestibility of the fibrous fractions of the diet. Thus, the digestibility of neutral detergent fiber (NDF) and acid detergent fiber (ADF) were significantly higher in diets containing sugar beet pulp and sweet potato tops than those of all other diets of mango bean hay and rice straw. Battaa et al. (2013) concluded that dietary acid detergent fiber (ADF) at level of $23.55 \%$ could be recommended to obtain the best growth performance growing rabbits. Along the same line, Safwat (2010) found that rabbit fed the control diet has significantly the highest value of feed intake, whereas the lowest was recorded by those received barley and Alfalfa; barley and berseem hay; barley and faba bean straw; corn-cob meal and Alfalfa as well as corn-cob meal and berseem hay respectively. Also, Sarhan (2005) showed that the live body weight at 9 weeks for rabbits fed diets contained pea vines hay and pea pods hulls at levels up to $30 \%$ were in significant higher than those of the control diet. With advancing age up to 13 weeks, the live body weight of rabbits received the same diet were significantly higher than that of the control. On the other hand, Genedy et al. (2000) revealed that body weight gain of rabbits fed dried water melon by - product at levels of $0,4,8$ and $12 \%$ at 12 weeks of age did not significantly differ than those received the control diet. This result is well correlated with finding of Al-Shanti (2003) who found no significant differences in live body weight and weight gain among rabbits fed $5,10,15$ and $20 \%$ olive cake meal with 
using $0,6.25,12.5,18.75$ and $25 \%$ of wheat screening by-product in rabbits diet, Ismail (2004) found that there were no significant differences in body weight and weight gain. Soliman et al. (2005) reported that rabbits fed diet with 14 and $12 \% \mathrm{CF}$ had higher feed intake than those fed diet contained $10 \% \mathrm{CF}$, while feed conversion ratio was not significantly affected by dietary fiber level. The objective for this study is to determine the chemical composition of PPM and consequently their effects on the productive performance and economic efficiency of the two lines of growing rabbits were also studied.

\section{MATERIALS AND METHODS}

The experimental work was conducted at Rabbit Production Unit, Poultry Production Department, Faculty of Agriculture, Alexandria University, Egypt, throughout the experimental growth period from November (2013) up to January, (2014). Fresh potato peal obtained from a commercial potato chip making units, were washed with tap water and dried by using sunshine. The dried peel was ground in a multi mill to obtain fine powder. Then, the meal kept in plastic bags until the preparation of experimental diets. Sample of the prepared potato peels meal was taken for estimating its chemical composition according to A.O.A.C. (2000). A total number of three hundred weaned rabbits, fifty-fifty between Alex. and V-lines, at 4 weeks of age having nearly equal live weights according to their guide line were used in this study. Rabbits of each line were individually weighted, randomly distributed into five equal groups (30 each treatment). Each group divided into ten replicates, each replicate contains 3 animals. Rabbits individually were housed in galvanized wire caged $(30 \times 35 \times 40$ $\mathrm{cm})$. Each cage was equipped with an automatic drinker stainless steel nipple and a manual feeder allowing recording individually feed intake for each rabbit. All animals were kept under the same managerial, hygienic and environmental conditions throughout the experimental growth periods. A photoperiod of 14 to 16 hours of day light was also provided throughout the experimental periods. Feed and clean fresh water were offered $a d$ libitum. The feeding period was extended for 10 weeks of age.

Five experimental diets were formulated and pelleted to cover the nutrient requirements of rabbits according to NRC (1994) and De Blas and Mateos (1998) as shown in Table (1). In that, graded levels of the ground potato peel meal were incorporated into experimental diets which were classified as follow:

Diet (1): Basal diet without replacement and served as control diet.

Diet (2): Basal diet replaced PPM at level $5 \%$ of dietary fiber.

Diet (3): Basal diet replaced PPM at level $10 \%$ ofdietary fiber.

Diet (4): Basal diet replaced PPM at level 15 of dietary fiber.

Diet (5): Basal diet replaced PPM at level $20 \%$ dietary fiber.

All diets had nearly iso-nutritive value but differ in their components according to the purpose study. The performance parameter included live body weight and feed consumption which were weekly determined and then weight gain and feed conversion ratio were calculated. At the end of the experimental period, the economic efficiency of studied experimental diets was calculated according to the local market price of ingredient and rabbit live body weight as following:

Net revenue $=$ total revenue - total feed cost.

Economic efficiency $(\%)=$ net revenue/ total feed cost $\%$.

Statistical analysis:

Collected data were subjected to statistical analyses by using the international software 
program (SAS, 2003). The application of the least significant ranges among different treatment means was done according to Duncan (1955).

The following statistical model was used:

$\mathrm{Y}_{\mathrm{ijk}}=\mu+\mathrm{L}_{\mathrm{i}}+\mathrm{T}_{\mathrm{j}}+\mathrm{LT}_{\mathrm{ij}}+\mathrm{e}_{\mathrm{ijk}}$

Where:

$\mathrm{Y}_{\mathrm{ijk}} \quad=$ The observation of the parameter measured،

$\mu=$ The overall mean،

$\mathrm{L}_{\mathrm{i}} \quad=$ The effect of line،

$\mathrm{T}_{\mathrm{j}} \quad=$ The effect of dietary treatment, $\mathrm{j}$

$=(1,2,3,4,5)$

$\mathrm{LT}_{\mathrm{ij}}=$ Interaction between lines and treatment.

$\mathrm{e}_{\mathrm{ijk}} \quad=$ The experimental random error.

\section{RESULTS AND DISCUSSION}

\section{Chemical Composition of PPM:}

Determined analysis on DM basis of studied materials as a dietary fiber is listed in Table (2). It is clearly shows that dry matter of potato peel meal had an intermediate score $(89.9 \%)$ between that of wheat bran and Alfalfa hay which being 88 and $90 \%$, respectively. However, dry matter of potato peel had a higher percentage compared with that of wheat bran. The opposite was true with organic matter, where the tested material had an approximately equal percentage $92 \%$ with that of Alfalfa hay and lower value than that of wheat bran which being $94 \%$.

Inspection in data, it is clearly shows that potato peel meal contained $14 \%$ crude protein compared with those of wheat brain and Alfalfa which being 15 and $15.5 \%$, respectively. In connection, result of crude protein was equal to that obtained by Omer et al. (2011). On the other hand, values of crude fiber and nitrogen free extract of the tested material fall within ranges of wheat brain and Alfalfa hay (15.6 vs. 11.0 or 25 and 57.1 vs. 64.0 or 49.5 ), respectively. Concerning the ether extract and ash content of potato peel meal, it were higher than those of other studied materials. Similar results were attained with values of gross energy and digestible energy which being 4296.85 vs. 1300 or $3950 \mathrm{Kcal} / \mathrm{kg}$ DM and 2574.8 vs. 2550 or $2175 \mathrm{Kcal} / \mathrm{kg}$ $\mathrm{DM}$, respectively. So it is of a great importance to note that nutrient substances of potato peel meal are of relatively suitable values to its rank as a dietary fiber for growing rabbit's.

\section{Productive Performance of the Experimental Groups:}

Data of tables (3 and 4), clearly shows that Alexandria rabbits recorded the heaviest weight and gain compared with those of V-line throughout the entire experimental ages up to 10 weeks. This may be due to the wide genetic variation between rabbits of the two studied lines (El-Raffa, 2007; Khamis, 2014). Irrespective of lines differences, it is obvious that rabbits fed diet contained $10 \%$ potato peel meal recorded the heaviest weight and gain compared with those received experimental diets of other studied levels through the most studied ages. This trend did not continue but it followed a reduction trend when animal fed either of diets contained 15 or $20 \%$ potato peel meal. The reported improvement in final live body weight with increasing dietary potato peel containing experimental diet up to $10 \%$ could be partially attributed to its effect on suppression of pathogenic organisms in the rabbit cecum (Omer et al., 2011). On the other hand, the significant decrease in body weight and gain with increasing the tested material may be due to increase neutral detergent fiber, acid detergent lignin and hemicelluloses contents in experimental diets. Generally, the best weight and gain were obtained from Alex. rabbits when fed diet of $10 \%$ potato peel being 2010.74 and $1329.44 \mathrm{~g}$. followed by those of $\mathrm{V}$-line received diet containing $5 \%$ potato peel which being 1959.73 and $1296.27 \mathrm{~g}$ as shown in Tables (4 and 6). In accordance with the present results, Elazab et al. (2011) reported that the highest growth rate came with rabbits 
on the $15 \%$ dried tomato pomace, while the lowest growth rate came with rabbits fed $20 \%$ the same tested material. So, the down word trend observed from diet contained 15 or $20 \%$ potato peel meal could be attributed to the effect of ant nutritional factors becoming more pronounced as the quantity of the test ingredient increased (Eka, 1977; Osagie, 1998).

Rabbits of V-Line consumed more diet compared with those of Alex- Line by about $5.13 \%$ through the whole period of (4-10) weeks as shown in Table (7). This result supported by the previous findings of Khamis (2014) who reported that animal of Alex. line consumed the least amount of diet as compared with those of V-Line but without significant. Also, it is obvious that increasing inclusion levels of tested ingredient up to $15 \%$, irrespective of lines difference, resulted to increase the amount of feed consumed by about $0.93 \%$ over that of the control diet but without significant. The opposite was recorded as feeding diet of $20 \%$ potato peel meal, where feed intake was significantly decreased compared with the studied inclusion levels. However, the highest amount of feed intake was recorded by V. rabbits at level of $5 \%$ being $3556.2 \mathrm{~g}$. whereas; the lowest amount was recorded by Alex. rabbits which being 3304.6 at $5 \%$ followed by $3331.4 \mathrm{~g}$ for $10 \%$ tested material (Tables 8). On the other hand, feed conversion ratio expressed as feed intake $(\mathrm{kg}) / \mathrm{unit}$ weight gain $(\mathrm{kg})$ in Table (9) clearly shows that Alex. rabbits had the best feed conversion ratio compared with those of V-Line over all studied period from 4 to 10 weeks. Along the same line, rabbits received diets of 5 or $10 \%$ dietary potato peel gave statistically similar values compared with those fed the control diet. Meanwhile, rabbits fed diet contained $10 \%$ tested ingredient recorded the superior score compared with rabbits of the other treatments. This trend did not continue but it significantly decreased as increasing inclusion level of dietary potato peel. It may be attributed to poor nutrient utilization as results of anti-nutrients as the quantity of the tested ingredient increased in the diet. Moreover, Offor et al. (2011) reported that potato contain solanine and chaconine which are more concentration in the peel which cause gastrointestinal upsets and neurological disorders (Osagie and Eka, 1988). Along the same line, Fapohunda et al. (2008), Adeyemi et al. (2010) and Aboh et al. (2013) observed the tendency trend when increasing inclusion levels of pineapple peel increased up to $40 \%$. So, it is clear from Table (10) that the best feed conversion ratio was obtained by Alex. rabbits fed experimental diet contained $10 \%$ tested ingredient, whereas those of V-Line gave the best score as feeding inclusion level 5\%. From this finding, it might be concluded that the experimental diet contained 5\% and $10 \%$ dietary potato peels seems more efficient for growing rabbits of $\mathrm{V}$. and Alex. lines as indicated by the presence of high gain as well as more beneficial growth rate, respectively.

\section{Economic Efficiency of dietary treatments:}

From the economic point of view the profitability, it is worth to note that the profitability of using dietary potato peel meal in rabbit diets depends on the price of tested diets and growth performance of rabbits fed these diets. Inspection in Table (11), it is obvious that costing of one $\mathrm{kg}$ feed (LE) was gradually decreased as increasing dietary potato peel containing experimental diets. So, the dietary inclusion levels of tested ingredient 5 and $10 \%$ scored less feed cost per $\mathrm{kg}$ body gain, being 7.07 and 7.15 (LE) for V. rabbits and 6.57 and 6.58 (LE) for those of Alex. Line, when compared with the other dietary treatments inclusion through growth period from 4 to 10 weeks old. Meanwhile, the least values were attained with Alex. rabbits in this respect. Consequently, by gradually increasing the rate of PPM as a 
replacement of wheat bran and Alfalfa up to $10 \%$, the total revenue, net revenue, economic efficiency and relative economic efficiency increased. The opposite was true with using the tested ingredient up to 15 or $20 \%$, respectively. It may be due to the low price of potato peel meal and the improvement of growth performance of growing rabbits for studied treatments compared to the control groups.

Generality, the relative economic efficiency (REE) of the different formulated diets as affected by dietary treatments of 5 and $10 \%$ were highly by 8.9 and $16.1 \%$ over the corresponding of the control group for Alex. Rabbits and followed by 7.2 and $5.5 \%$ over those the control group of V. rabbits, respectively. In connection, a result of Khamis (2014) is in agreement with our finding where the superiority rate of the economic efficiency was attained with Alex-rabbits compared with those of V-line. Along the same line, Sarhan (2005) and Safwat (2010) indicated that using faba bean straw as a source of fiber instead of Alfalfa and berseem hay gave the superiority rate in this respect. 
Table (1): Composition and chemical analysis of experimental diets.

\begin{tabular}{|c|c|c|c|c|c|}
\hline \multirow{2}{*}{ Ingredient, \% } & \multicolumn{5}{|c|}{ Potato peel meal replacement levels, $\%$} \\
\hline & $\mathbf{0 . 0}$ & 5 & 10 & 15 & 20 \\
\hline Yellow corn & 7.00 & 7.00 & 7.00 & 7.00 & 7.00 \\
\hline Wheat bran & 20.00 & 17.50 & 15.00 & 12.50 & 10.00 \\
\hline Barely & 18.30 & 18.20 & 18.00 & 17.90 & 17.80 \\
\hline Alfalfa & 22.50 & 20.00 & 17.50 & 15.00 & 12.50 \\
\hline Soybean meal, $44 \%$ & 18.20 & 18.40 & 18.50 & 18.60 & 18.70 \\
\hline Straw & 8.00 & 8.00 & 8.00 & 8.00 & 8.00 \\
\hline Potato Peel & - & 5.00 & 10.00 & 15.00 & 20.00 \\
\hline Di-calcium phosphate & 1.9 & 1.7 & 1.7 & 1.7 & 1.7 \\
\hline Limestone & 0.2 & 0.3 & 0.4 & 0.4 & 0.4 \\
\hline Methionine & 0.15 & 0.15 & 0.15 & 0.15 & 0.15 \\
\hline Lysine & 0.05 & 0.05 & 0.05 & 0.05 & 0.05 \\
\hline Salt & 0.4 & 0.4 & 0.4 & 0.4 & 0.4 \\
\hline Premix* & 0.3 & 0.3 & 0.3 & 0.3 & 0.3 \\
\hline Molasses & 3.00 & 3.00 & 3.00 & 3.00 & 3.00 \\
\hline Total & 100 & 100 & 100 & 100 & 100 \\
\hline Determined analyses on DM & & & & & \\
\hline basis: & 17.50 & 17.43 & 17.48 & 17.45 & 17.51 \\
\hline$\overline{\text { Crude }}$ protein $\%$ & 13.40 & 13.30 & 13.30 & 13.20 & 13.10 \\
\hline Crude fiber $\%$ & 2.10 & 2.20 & 2.30 & 2.20 & 2.20 \\
\hline Ether extract \% & & & & & \\
\hline Calculated values: & 2545 & 2544 & 2543 & 2546 & 2547 \\
\hline Digestible energy $(\mathrm{kcal} / \mathrm{kg}) * *$ & 7.20 & 7.08 & 7.07 & 7.02 & 7.01 \\
\hline Ash \% & 145.42 & 145.95 & 145.48 & 145.90 & 145.45 \\
\hline $\mathrm{DE} / \mathrm{P}$ & 0.92 & 0.91 & 0.92 & 0.94 & 0.93 \\
\hline Calcium $\%$ & 0.60 & 0.60 & 0.60 & 0.60 & 0.60 \\
\hline Phosphorus available $\%$ & 0.40 & 0.40 & 0.40 & 0.40 & 0.40 \\
\hline $\begin{array}{l}\text { Methionine \% } \\
\text { Lysine \% }\end{array}$ & 0.91 & 0.91 & 0.91 & 0.91 & 0.91 \\
\hline
\end{tabular}

* Vitamin-mineral premix provide per kg of diet vit. A, 13.340 iu; vit. D3, 2680. i.u; vit. E, 10 i.u; vit. K, $2.68 \mathrm{mg}$; Calcium pantothenate, $10.68 \mathrm{mg}$; vit. B12, $0.022 \mathrm{mg}$; folic acid, $0.668 \mathrm{mg}$; choline chloride, $400 \mathrm{mg}$; chlorotetracycline, $26.68 \mathrm{mg}$; manganese, $133.34 \mathrm{mg}$; iron, 66.68 $\mathrm{mg}$; zinc, $53.34 \mathrm{mg}$; copper, $3.2 \mathrm{mg}$, iodine, $1.86 \mathrm{mg}$; cobalt, $0.268 \mathrm{mg}$, selenium, $0.108 \mathrm{mg}$.

** Calculated DE $(\mathrm{Kcal} / \mathrm{kg})=\mathrm{TDN} \times 44.3($ Schneider and Flatt, 1975). 
Table (2): Chemical Composition of Dried potato peel, Wheat bran, and Alfalfa hay as an ingredient in rabbits diets.

\begin{tabular}{|l|c|c|c|}
\hline Item & Potato peels & Wheat bran* & Alfalfa hay* \\
\hline Chemical analyses (\%) & \multicolumn{3}{|l|}{} \\
Dry matter (DM) & 89.90 & 88.00 & 90.00 \\
\hline Chemical analyses on DM basis & 91.90 & 94.00 & 92.00 \\
\hline Organic matter (OM) & 14.00 & 15.00 & 15.50 \\
Crude protein (CP) & 15.60 & 11.00 & 25.00 \\
Crude fiber (CF) & 5.20 & 4.00 & 2.00 \\
Ether extract (EE) & 57.10 & 64.00 & 49.50 \\
Nitrogen free extract (NFE) & 8.10 & 6.00 & 8.00 \\
Ash & 4296.85 & 1300.00 & 3950.00 \\
Gross energy (kcal/kg DM) & 2574.80 & 2550.00 & 2175.00 \\
Digestible energy (kcal/kg DM) & \multicolumn{3}{|l}{} \\
\hline
\end{tabular}

*Source : NRC 1994 
Table (3): The main effect of two lines of growing rabbits fed different inclusion levels of Potato Peel meal on live body weight during experimental growth ages.

\begin{tabular}{|c|c|c|c|c|c|c|c|}
\hline \multirow[b]{2}{*}{ Items } & \multicolumn{7}{|c|}{ Live Body weight (g./ rabbit) } \\
\hline & 4 wks & 5 wks & $6 \mathrm{wks}$ & 7 wks & 8 wks & 9 wks & $10 \mathrm{wks}$ \\
\hline \multicolumn{8}{|l|}{ Lines } \\
\hline$\overline{\mathrm{V} .}$ & $658.85 \pm 12.60$ & $804.66 \pm 16.18$ & $961.38 \pm 17.06$ & $1145.65^{b} \pm 20.79$ & $1341.32^{b} \pm 21.46$ & $1564.38^{b} \pm 23.52$ & $1805.23^{b} \pm 22.84$ \\
\hline Alex. & $675.90 \pm 11.72$ & $823.69 \pm 13.47$ & $990.97 \pm 15.91$ & $1190.76^{\mathrm{a}} \pm 20.14$ & $1390.16^{\mathrm{a}} \pm 21.02$ & $1623.89^{\mathrm{a}} \pm 22.12$ & $1859.79^{\mathrm{a}} \pm 22.07$ \\
\hline \multicolumn{8}{|l|}{$\begin{array}{l}\text { Dietary Potato Peel } \\
\text { meal \% }\end{array}$} \\
\hline 0.0 & $668.06 \pm 20.65$ & $825.51 \pm 25.25$ & $990.59^{\mathbf{b}} \pm 26.35$ & $1189.95^{b} \pm 36.07$ & $1395.19^{\mathbf{b}} \pm 35.74$ & $1648.84^{\mathrm{a}} \pm 38.31$ & $1900.01^{\mathbf{b}_{ \pm}} \pm 37.55$ \\
\hline 5 & $666.21 \pm 15.85$ & $828.06 \pm 18.08$ & $1024.55^{\mathrm{a}} \pm 22.46$ & $1226.30^{\mathrm{a}} \pm 24.76$ & $1441.50^{\mathrm{a}} \pm 24.98$ & $1688.28^{\mathrm{a}} \pm 27.66$ & $1955.33^{\mathrm{a}} \pm 31.59$ \\
\hline 10 & $666.73 \pm 18.55$ & $827.45 \pm 24.31$ & $994.90^{\mathbf{b}} \pm 28.34$ & $1201.65^{\mathbf{a b}} \pm 39.25$ & $1418.54^{\mathrm{ab}_{ \pm}} 37.06$ & $1679.76^{\mathrm{a}} \pm 38.25$ & $1946.86^{a} \pm 36.77$ \\
\hline 15 & $673.71 \pm 16.52$ & $826.43 \pm 18.09$ & $1007.39^{\mathrm{a}} \pm 19.17$ & $1201.24^{\mathbf{a b}_{ \pm}} \pm 19.44$ & $1390.36^{\mathbf{b}} \pm 23.32$ & $1591.69^{\mathbf{b}} \pm 25.11$ & $1802.85^{\mathrm{c}} \pm 28.51$ \\
\hline 20 & $662.16 \pm 24.09$ & $763.42 \pm 30.11$ & $863.46^{\mathbf{c}} \pm 31.97$ & $1021.87^{\mathrm{c}} \pm 38.23$ & $1182.72^{\mathrm{c}} \pm 41.31$ & $1362.11^{\mathrm{c}} \pm 45.27$ & $1557.51^{\mathrm{d}} \pm 42.23$ \\
\hline \multicolumn{8}{|l|}{ Significance } \\
\hline Lines & NS & NS & NS & $*$ & $*$ & $*$ & $*$ \\
\hline $\begin{array}{l}\text { Dietary Potato Peel } \\
\text { meal }\end{array}$ & NS & NS & $*$ & $*$ & $* *$ & $* *$ & $*$ \\
\hline
\end{tabular}


Table (4): The interaction effect between two lines of growing rabbits by different inclusion levels of Potato Peel meal on live body weight during experimental growth ages.

\begin{tabular}{|c|c|c|c|c|c|c|c|}
\hline \multirow{2}{*}{ Items } & \multicolumn{7}{|c|}{ Live body weight (g./ rabbit) } \\
\hline & 4 wks & 5 wks & $6 \mathrm{wks}$ & $7 \mathrm{wks}$ & 8 wks & 9 wks & 10 wks \\
\hline V-line $\times$ control & $660.33 \pm 29.54$ & $809.43 \pm 39.33$ & $969.69^{b} \pm 40.23$ & $1157.10^{c} \pm 52.29$ & $1358.54^{b} \pm 51.05$ & $1616.39^{c} \pm 57.04$ & $1887.46^{\mathrm{c}} \pm 54.53$ \\
\hline $\begin{array}{l}\text { V-line } \times 5 \% \text { Potato } \\
\text { Peel }\end{array}$ & $663.46 \pm 27.96$ & $839.16 \pm 30.61$ & $1033.52^{\mathrm{a}} \pm 34.07$ & $1236.85^{a} \pm 39.09$ & $1458.91^{\mathrm{a}} \pm 37.75$ & $1694.15^{b} \pm 41.07$ & $1959.73^{b} \pm 44.89$ \\
\hline $\begin{array}{l}\text { V-line } \times 10 \% \text { Potato } \\
\text { Peel }\end{array}$ & $652.16 \pm 26.29$ & $819.00 \pm 36.75$ & $975.89^{b} \pm 39.92$ & $1167.99^{c} \pm 52.47$ & $1374.95^{\mathbf{b}} \pm 47.07$ & $1626.70^{\mathrm{c}} \pm 47.82$ & $1882.98^{c} \pm 45.43$ \\
\hline $\begin{array}{l}\text { V-line } \times 15 \% \text { Potato } \\
\text { Peel }\end{array}$ & $666.90 \pm 27.39$ & $808.00 \pm 29.40$ & $990.40^{b} \pm 29.49$ & $1192.60^{b} \pm 33.06$ & $1384.91^{b} \pm 39.58$ & $1584.84^{\mathrm{d}} \pm 42.52$ & $1804.87^{\mathrm{d}} \pm 42.18$ \\
\hline $\begin{array}{l}\text { V-line } \times 20 \% \text { Potato } \\
\text { Peel }\end{array}$ & $651.40 \pm 31.28$ & $747.71 \pm 44.33$ & $837.43^{d} \pm 45.46$ & $973.72^{d} \pm 43.33$ & $1129.32^{d} \pm 48.80$ & $1299.85^{\mathrm{f}} \pm 50.81$ & $1491.14^{\mathbf{f}} \pm 54.10$ \\
\hline Alex-line $\times$ control & $675.80 \pm 15.41$ & $841.60 \pm 18.38$ & $1011.50^{\mathrm{a}} \pm 20.81$ & $1222.81^{\mathrm{a}} \pm 21.88$ & $1431.84^{\mathrm{a}} \pm 24.47$ & $1681.29^{b} \pm 26.74$ & $1912.56^{b} \pm 32.74$ \\
\hline $\begin{array}{l}\text { Alex-line } \times 5 \% \text { Potato } \\
\text { Peel }\end{array}$ & $668.96 \pm 29.35$ & $816.96 \pm 32.03$ & $1015.58^{a} \pm 34.70$ & $1215.76^{\mathrm{a}} \pm 50.55$ & $1424.09^{\mathrm{a}} \pm 50.96$ & $1682.42^{\mathbf{b}} \pm 52.01$ & $1950.93^{\mathbf{b}} \pm 52.20$ \\
\hline $\begin{array}{l}\text { Alex-line } \times 10 \% \text { Potato } \\
\text { Peel }\end{array}$ & $681.30 \pm 26.36$ & $835.91 \pm 31.62$ & $1013.91^{\mathrm{a}} \pm 38.47$ & $1235.32^{\mathrm{a}} \pm 59.09$ & $1462.14^{\mathrm{a}} \pm 57.44$ & $1732.82^{\mathrm{a}} \pm 59.60$ & $2010.74^{\mathrm{a}} \pm 58.22$ \\
\hline $\begin{array}{l}\text { Alex-line } \times 15 \% \text { Potato } \\
\text { Peel }\end{array}$ & $680.53 \pm 18.89$ & $844.86 \pm 21.03$ & $1024.39^{\mathrm{a}} \pm 24.79$ & $1209.89^{b} \pm 20.05$ & $1396.62^{b} \pm 23.99$ & $1598.54^{\text {cd }} \pm 23.88$ & $1800.84^{d} \pm 34.25$ \\
\hline $\begin{array}{l}\text { Alex-line } \times 20 \% \text { Potato } \\
\text { Peel }\end{array}$ & $672.93 \pm 37.09$ & $779.13 \pm 41.47$ & $889.50^{\mathbf{c}} \pm 44.58$ & $1070.02^{\mathrm{e}} \pm 56.03$ & $1236.13^{\mathrm{c}} \pm 59.83$ & $1424.38^{\mathrm{e}} \pm 58.87$ & $1623.89^{\mathrm{e}} \pm 54.65$ \\
\hline Significance & NS & NS & $* *$ & $* *$ & $* *$ & $* *$ & $* * *$ \\
\hline
\end{tabular}

$* *=$ Significant at $\mathrm{p} \leq 0.01 \quad$ N.S $=$ not significant

Different letters $(\mathrm{a}, \mathrm{b}, \mathrm{c}, \mathrm{d}, \mathrm{e})$ in the same column indicate significant differences $\mathrm{p} \leq 0.05$ 
Table (5): The main effect of two lines of growing rabbits fed different inclusion levels of Potato Peel meal on live body weight gain during experimental growth periods.

\begin{tabular}{|c|c|c|c|c|c|c|c|}
\hline \multirow{2}{*}{ Items } & \multicolumn{7}{|c|}{ Live body weight gain (g./week) } \\
\hline & (4-5) wks & $(5-6)$ wks & (6-7) wks & (7-8) wks & (8-9) wks & $(9-10)$ wks & (4-10) wks \\
\hline Lines & & & & & & & \\
\hline V. & $145.80 \pm 7.66$ & $147.72^{b} \pm 5.60$ & $184.26^{\mathbf{b}} \pm 9.54$ & $195.68 \pm 5.79$ & $223.06^{\mathbf{b}} \pm 6.68$ & $240.85 \pm 6.81$ & $1146.38^{\mathbf{b}} \pm 16.09$ \\
\hline Alex. & $147.78 \pm 5.61$ & $167.28^{\mathrm{a}_{ \pm}}+5.85$ & $199.78^{a} \pm 10.49$ & $199.40 \pm 6.30$ & $233.71^{a} \pm 5.77$ & $235.90 \pm 6.57$ & $1183.88^{\mathrm{a}} \pm 17.87$ \\
\hline $\begin{array}{l}\text { Dietary Potato Peel } \\
\text { meal\% }\end{array}$ & & & & & & & \\
\hline 0.0 & $157.45^{b_{ \pm}} \pm 12.60$ & $165.08^{b_{ \pm}}+7.38$ & $199.36^{\mathbf{a}} \pm 19.00$ & $205.23^{\mathrm{a}} \pm 8.61$ & $253.63^{a} \pm 10.65$ & $251.17^{\mathbf{b}_{ \pm} 9.68}$ & $1231.92^{\mathbf{b}_{ \pm}} 27.79$ \\
\hline 5 & $161.85^{\mathrm{a}} \pm 11.56$ & $196.49 a 9.73$ & $201.75^{a} \pm 8.25$ & $215.19^{\mathrm{a}} \pm 8.54$ & $246.78^{b} \pm 12.19$ & $267.04^{\mathrm{a}} \pm 11.21$ & $1289.12^{\mathrm{a}} \pm 24.13$ \\
\hline 10 & $160.72^{\mathrm{a}} \pm 13.46$ & $167.44^{b_{ \pm}}{ }^{9} .48$ & $206.75^{a} \pm 21.66$ & $216.89^{\mathrm{a}} \pm 9.98$ & $261.21^{\mathrm{a}} \pm 9.25$ & $267.10^{\mathrm{a}} \pm 9.23$ & $1280.12^{\mathrm{a}} \pm 26.68$ \\
\hline 15 & $152.69^{c} \pm 15.94$ & $180.96^{\mathrm{a}} \pm 8.87$ & $193.85^{b_{ \pm}}{ }_{8.41}$ & $189.54^{\mathbf{b}} \pm 7.77$ & $200.92 c_{ \pm}{ }_{8.57}$ & $211.16^{c} \pm 8.36$ & $1129.14^{c} \pm 26.70$ \\
\hline 20 & $101.25^{\mathrm{d}} \pm 10.6$ & $100.04^{c^{c}} \pm 8.71$ & $158.40^{c} \pm 15.71$ & $160.85^{\mathrm{c}} \pm 9.28$ & $197.39^{c_{ \pm} 9.64}$ & $195.40^{d_{ \pm}}+6.69$ & $895.35^{\mathrm{d}} \pm 25.90$ \\
\hline Significance & & & & & & & \\
\hline Lines & & & & & & & \\
\hline Dietary Potato Peel & NS & $* * *$ & $* *$ & NS & *** & NS & *** \\
\hline meal & $* * *$ & $* * *$ & $* * *$ & ** & $* * *$ & $* * *$ & $* * *$ \\
\hline
\end{tabular}

$* *=$ Significant at $\mathrm{p} \leq 0.01 \quad * * *=$ Significant at $\mathrm{p} \leq 0.001 \quad \mathrm{~N} . \mathrm{S}=$ not significant

Different letters $(\mathrm{a}, \mathrm{b}, \mathrm{c}, \mathrm{d})$ in the same column indicate significant differences $\mathrm{p} \leq 0.05$. 
Table (6): The interaction effect between two lines of growing rabbits by different inclusion levels of Potato Peel meal on live body weight gain during experimental growth periods.

\begin{tabular}{|c|c|c|c|c|c|c|c|}
\hline \multirow{2}{*}{ Items } & \multicolumn{7}{|c|}{ Live body weight gain (g./week) } \\
\hline & (4-5) wks & $(5-6)$ wks & $(6-7)$ wks & $(7-8)$ wks & (8-9) wks & $(9-10)$ wks & $(4-10)$ wks \\
\hline V-line $\times$ control & $149.10^{b_{ \pm}} \pm 12.29$ & $160.26^{c^{c}} \pm 9.83$ & $187.41^{b_{ \pm}} \pm 25.75$ & $201.44^{b} \pm 19.67$ & $257.85^{b} \pm 17.24$ & $271.07^{\mathrm{a}} \pm 9.87$ & $1227.13^{c} \pm 21.45$ \\
\hline V-line $\times 5 \%$ Potato Peel & $175.70^{\mathrm{a}} \pm 11.93$ & $194.36^{\mathrm{a}} \pm 11.57$ & $203.33 \mathrm{a} \pm 11.96$ & $222.06^{\mathrm{a}} \pm 10.14$ & $235.24^{\mathrm{c}} \pm 10.95$ & $265.58 \mathrm{a}_{ \pm} \pm 12.44$ & $1296.27^{b_{ \pm}} \pm 22.74$ \\
\hline $\begin{array}{l}\text { V-line } \times 10 \% \text { Potato } \\
\text { Peel }\end{array}$ & $166.83^{\mathrm{a}} \pm 23.52$ & $156.89^{c} \pm 10.96$ & $192.10^{\mathrm{a}} \pm 11.91$ & $206.96^{\mathbf{b}} \pm 26.38$ & $251.75^{b} \pm 16.77$ & $256.28^{\mathbf{b}_{ \pm}}+14.73$ & $1230.81^{\mathbf{c}} \pm 19.85$ \\
\hline V-line $\times 15 \%$ Potato Peel & $141.06^{\mathbf{b}} \pm 6.78$ & $182.40^{\mathbf{a b}} \pm 11.95$ & $202.20^{a} \pm 11.96$ & $192.35^{\mathrm{c}} \pm 10.31$ & $199.93 \mathrm{~d}_{ \pm 1} 1.03$ & $220.03^{c} \pm 10.46$ & $1137.97 \mathrm{~d}_{ \pm} 19.75$ \\
\hline V-line $\times 20 \%$ Potato Peel & $96.31^{c} \pm 12.56$ & $89.72 \mathrm{e}_{ \pm} \mathrm{e}_{9.14}$ & $136.29^{c} \pm 16.53$ & $155.60^{\mathrm{e}} \pm 12.04$ & $170.53^{\mathbf{f}_{ \pm}}+6.68$ & $191.29^{d} \pm 16.53$ & $839.74^{\mathbf{f}_{ \pm}}+17.35$ \\
\hline Alex-line $\times$ control & $165.80 a \pm 9.34$ & $169.90^{c} \pm 13.21$ & $211.31 \mathrm{a} \pm 11.05$ & $209.03^{b} \pm 11.62$ & $249.41^{b_{ \pm}} \pm 11.52$ & $231.27^{\mathrm{c}} \pm 15.79$ & $1236.72^{c} \pm 20.70$ \\
\hline $\begin{array}{l}\text { Alex-line } \times 5 \% \text { Potato } \\
\text { Peel }\end{array}$ & $148.00^{b_{ \pm}}+14.67$ & $198.62^{\mathrm{a}} \pm 28.37$ & $200.18^{a} \pm 13.39$ & $208.33^{\mathbf{b}_{ \pm}} \mathbf{9 . 2 2}$ & $258.33^{\mathbf{b}_{ \pm}}+12.64$ & $268.51^{\mathrm{a}} \pm 14.45$ & $1281.97^{\mathbf{b}_{ \pm} 22.47}$ \\
\hline $\begin{array}{l}\text { Alex-line } \times 10 \% \text { Potato } \\
\text { Peel }\end{array}$ & $154.61^{\mathbf{a b}_{ \pm}}+34.64$ & $178.00^{\mathbf{b}_{ \pm}} 15.77$ & $221.41^{\mathrm{a}} \pm 12.92$ & $226.82^{\mathrm{a}} \pm 13.98$ & $270.68^{\mathrm{a}} \pm 10.71$ & $277.922^{a} \pm 9.37$ & $1329.44^{\mathrm{a}} \pm 24.78$ \\
\hline $\begin{array}{l}\text { Alex-line } \times 15 \% \text { Potato } \\
\text { Peel }\end{array}$ & $164.33^{\mathrm{a}} \pm 8.84$ & $179.53^{b_{ \pm}} \pm 9.16$ & $185.50^{\mathbf{b}_{ \pm 2} 3.64}$ & $186.73^{c} \pm 10.38$ & $201.92^{d_{ \pm}}{ }_{10.39}$ & $202.30^{d_{ \pm}}{ }_{14.36}$ & $1120.31^{d_{ \pm}}+19.45$ \\
\hline $\begin{array}{l}\text { Alex-line } \times 20 \% \text { Potato } \\
\text { Peel }\end{array}$ & $106.20^{c} \pm 12.10$ & $110.37^{d_{ \pm}}+11.06$ & $180.52^{\mathbf{b}_{ \pm}}+11.49$ & $166.11^{d} \pm 12.29$ & $188.25^{\mathrm{e}_{ \pm}}+14.08$ & $199.51^{d_{ \pm}}+13.06$ & $950.96^{e^{e}} \pm 16.23$ \\
\hline Significance & $* *$ & $* * *$ & $* * *$ & $*$ & $* * *$ & $* * *$ & $* * *$ \\
\hline
\end{tabular}

$*=$ Significant at $\mathrm{p} \leq 0.05 \quad * *=$ Significant at $\mathrm{p} \leq 0.01 \quad * * *=$ Significant at $\mathrm{p} \leq 0.001$

Different letters $(a, b, c, d, e)$ in the same column indicate significant differences $p \leq 0.0$ 
Table (7): The main effect of two lines of growing rabbits fed different inclusion levels of Potato Peel meal on feed intake during experimental growth periods.

\begin{tabular}{|c|c|c|c|c|c|c|c|}
\hline \multirow{2}{*}{ Items } & \multicolumn{7}{|c|}{ Feed Intake (g./week/rabbit) } \\
\hline & (4-5) wks & $(5-6)$ wks & $(6-7)$ wks & $(7-8)$ wks & (8-9) wks & (9-10) wks & (4-10) wks \\
\hline \multicolumn{8}{|l|}{ Lines } \\
\hline$\overline{\mathrm{V} .}$ & $338.60 \pm 3.99$ & $465.76 \pm 5.60$ & $439.08 \pm 8.83$ & $620.00 \mathrm{a} \pm 7.92$ & $700.52^{\mathrm{a}} \pm 5.49$ & $910.96^{\mathrm{a}} \pm 11.46$ & $3474.92 \mathrm{a} \pm 114.52$ \\
\hline \multirow{2}{*}{\multicolumn{8}{|c|}{$\frac{\text { Dietary Potato Peel }}{\text { meal\% }}$}} \\
\hline & & & & & & & \\
\hline$\overline{0.0}$ & $322.20 \pm 2.45$ & $473.90 \pm 2.34$ & $428.40 \pm 14.76$ & $600.40^{a} \pm 12.97$ & $679.80 \pm 14.64$ & $910.70^{a} \pm 18.46$ & $3415.40^{\mathbf{a}} \pm 99.45$ \\
\hline 5 & $340.50 \pm 3.42$ & $476.40 \pm 9.43$ & $430.90 \pm 15.01$ & $599.70^{a} \pm 8.71$ & $666.10 \pm 13.38$ & $916.80^{\mathrm{a}} \pm 18.23$ & $3430.40^{\mathrm{a}} \pm 89.45$ \\
\hline 10 & $320.10 \pm 3.72$ & $474.00 \pm 7.76$ & $429.80 \pm 10.62$ & $601.30^{a} \pm 19.45$ & $679.40 \pm 11.10$ & $916.00^{\mathrm{a}} \pm 18.26$ & $3420.60^{\mathrm{a}} \pm 94.47$ \\
\hline 15 & $334.80 \pm 8.24$ & $490.70 \pm 10.52$ & $435.70 \pm 12.02$ & $600.10^{a} \pm 22.05$ & $683.10 \pm 10.84$ & $902.70^{a} \pm 20.23$ & $3447.10^{\mathrm{a}} \pm 104.52$ \\
\hline 20 & $315.80 \pm 9.31$ & $464.60 \pm 13.03$ & $418.50 \pm 19.94$ & $561.90^{\mathbf{b}} \pm 18.46$ & $645.10 \pm 17.47$ & $831.50^{b_{ \pm}} \pm 25.67$ & $3237.40^{\mathbf{b}} \pm 101.85$ \\
\hline \multicolumn{8}{|l|}{ Significance } \\
\hline Lines & NS & NS & NS & $* * *$ & $* * *$ & $* *$ & $* * *$ \\
\hline Dietary Potato Peel meal & NS & NS & NS & $* *$ & NS & $* *$ & $* *$ \\
\hline
\end{tabular}

$* *=$ Significant at $\mathrm{p} \leq 0.01 \quad * * *=$ Significant at $\mathrm{p} \leq 0.001 \quad$ N.S $=$ not significant

Different letters $(a, b)$ in the same column indicate significant differences $p \leq 0.05$ 
Table (8): The interaction effect between two lines of growing rabbits by different inclusion levels of Potato Peel meal on feed intake during experimental growth periods.

\begin{tabular}{|c|c|c|c|c|c|c|c|}
\hline \multirow{2}{*}{ Items } & \multicolumn{7}{|c|}{ Feed Intake (g./week/rabbit) } \\
\hline & (4-5) wks & (5-6) wks & $(6-7)$ wks & $(7-8)$ wks & (8-9) wks & $(9-10)$ wks & (4-10) wks \\
\hline V-line $\times$ control & $334.00 \pm 1.22$ & $467.40^{b} \pm 0.74$ & $440.80 \pm 17.66$ & $630.00^{\mathrm{a}} \pm 11.44$ & $708.00^{\mathrm{a}} \pm 3.72$ & $926.00^{\mathrm{a}} \pm 21.90$ & $3506.20^{c} \pm 79.26$ \\
\hline V-line $\times 5 \%$ Potato Peel & $357.20 \pm 9.92$ & $477.40^{b} \pm 21.22$ & $450.00 \pm 31.65$ & $633.40^{\mathrm{a}} \pm 20.60$ & $706.40^{\mathrm{a}} \pm 16.56$ & $931.80^{\mathrm{a}} \pm 22.02$ & $3556.20^{\mathrm{a}} \pm 101.20$ \\
\hline V-line $\times 10 \%$ Potato Peel & $328.40 \pm 3.42$ & $465.80^{b} \pm 3.33$ & $440.40 \pm 17.50$ & $634.00^{a} \pm 10.67$ & $705.20^{\mathrm{a}} \pm 7.72$ & $936.00^{\mathrm{a}} \pm 19.28$ & $3509.80^{c} \pm 69.78$ \\
\hline V-line $\times 15 \%$ Potato Peel & $352.00 \pm 11.04$ & $474.60^{b} \pm 16.77$ & $440.00 \pm 18.99$ & $629.00^{a} \pm 18.06$ & $709.40^{\mathrm{a}} \pm 11.68$ & $931.40^{\mathrm{a}} \pm 21.90$ & $3536.40^{\mathbf{b}} \pm 98.10$ \\
\hline V-line $\times 20 \%$ Potato Peel & $321.40 \pm 3.04$ & $443.60^{c} \pm 4.72$ & $424.20 \pm 16.58$ & $573.60^{b} \pm 14.50$ & $673.60^{b} \pm 13.85$ & $829.60^{c} \pm 6.72$ & $3266.00^{\mathrm{g}} \pm 81.23$ \\
\hline Alex-line $\times$ control & $310.40 \pm 5.33$ & $480.40^{\mathbf{b}} \pm 14.46$ & $416.00 \pm 27.03$ & $570.80^{\mathbf{b}} \pm 11.42$ & $651.60^{\mathrm{c}} \pm 23.54$ & $895.40^{\mathbf{b}_{ \pm}}+30.17$ & $3324.60^{\mathbf{e}} \pm 98.23$ \\
\hline Alex-line $\times 5 \%$ Potato Peel & $323.80 \pm 3.55$ & $475.40^{b} \pm 4.01$ & $411.80 \pm 23.75$ & $566.00^{b} \pm 10.69$ & $625.80^{c} \pm 10.32$ & $901.80^{\mathbf{b}} \pm 31.33$ & $3304.60^{\mathbf{f}_{ \pm} 96.01}$ \\
\hline Alex-line $\times 10 \%$ Potato Peel & $311.80 \pm 4.04$ & $482.20^{b} \pm 15.04$ & $419.20 \pm 12.05$ & $568.60^{b} \pm 32.48$ & $653.60^{c} \pm 12.74$ & $896.00^{b} \pm 30.48$ & $3331.40^{\mathrm{e}} \pm 97.12$ \\
\hline Alex-line $\times 15 \%$ Potato Peel & $317.60 \pm 5.98$ & $506.80^{\mathrm{a}} \pm 9.36$ & $431.40 \pm 16.73$ & $571.20^{b} \pm 38.01$ & $656.80^{c} \pm 6.82$ & $874.00^{b} \pm 30.85$ & $3357.80^{\mathrm{d}} \pm 90.20$ \\
\hline Alex-line $\times 20 \%$ Potato Peel & $310.20 \pm 3.96$ & $485.60^{\mathbf{b}} \pm 17.48$ & $412.80 \pm 24.80$ & $550.20^{\mathbf{b}} \pm 15.64$ & $616.60^{\mathrm{c}} \pm 9.54$ & $833.40^{c} \pm 35.65$ & $3208.80^{h^{h}} \pm 93.75$ \\
\hline Significance & NS & $* *$ & NS & $* *$ & $* *$ & & \\
\hline
\end{tabular}

\footnotetext{
$* *=$ Significant at $\mathrm{p}<0.01$

N.S $=$ not significant
}

Different letters $(a, b, c, d, \ldots, h)$ in the same column indicate significant differences $p \leq 0.05$ 
Table (9): The main effect of two lines of growing rabbits fed different inclusion levels of Potato Peel meal on feed conversion ratio during experimental growth periods.

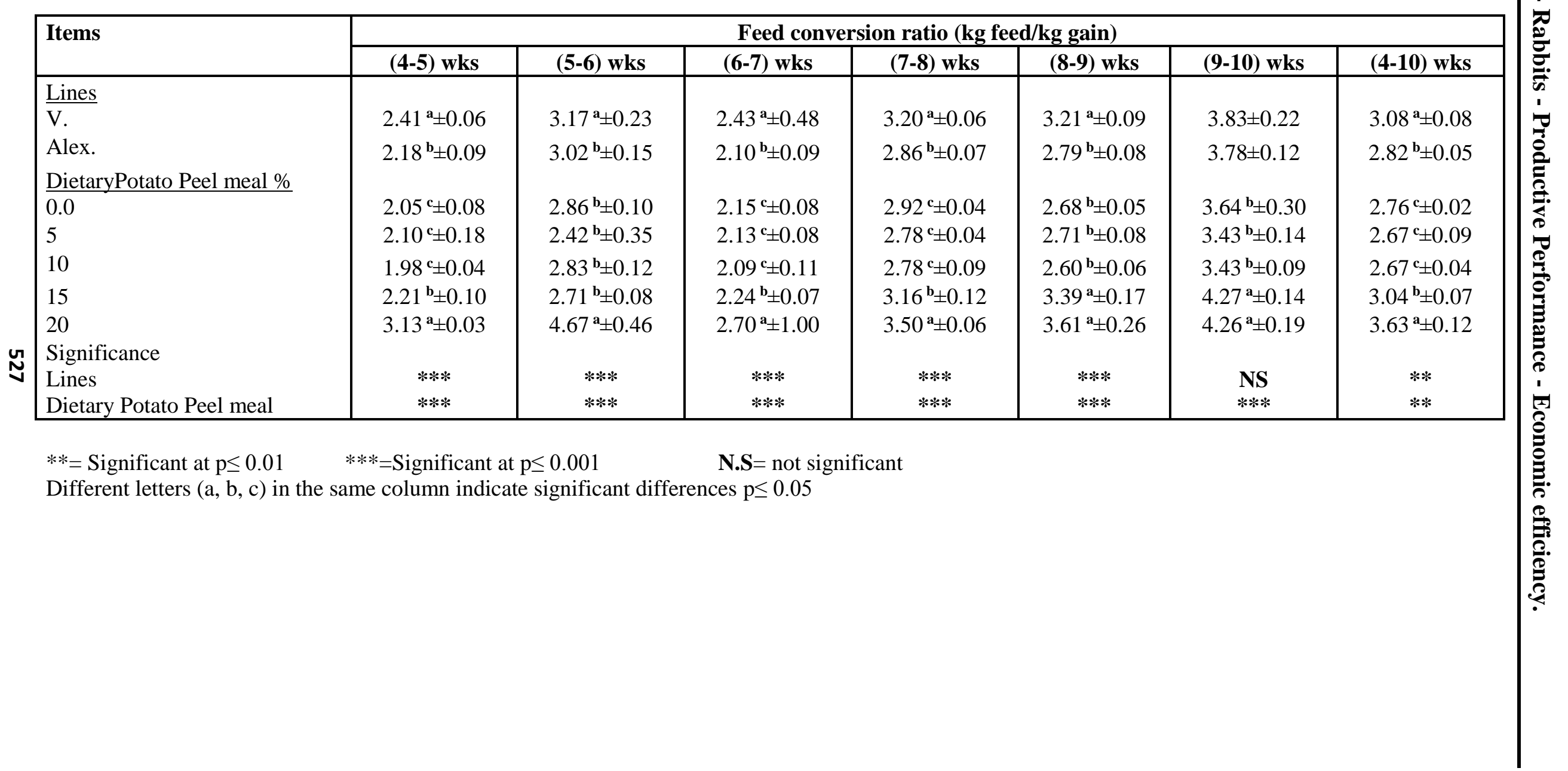


Table (10): The interaction effect between two lines of growing rabbits by different inclusion levels of Potato Peel meal on feed conversion ratio during experimental growth periods.

\begin{tabular}{|c|c|c|c|c|c|c|c|}
\hline \multirow{2}{*}{ Items } & \multicolumn{7}{|c|}{ Feed conversion ratio (kg feed/kg gain) } \\
\hline & (4-5) wks & (5-6) wks & (6-7) wks & $(7-8)$ wks & (8-9) wks & (9-10) wks & (4-10) wks \\
\hline V-line $\times$ control & $2.24^{\mathrm{c}} \pm 0.02$ & $2.91^{\mathrm{c}} \pm 0.05$ & $2.35^{\mathbf{b}} \pm 0.09$ & $3.12^{\mathbf{b}} \pm 0.04$ & $2.75^{\mathrm{c}} \pm 0.02$ & $3.41^{\mathrm{d}} \pm 0.14$ & $2.85^{\mathrm{c}} \pm 0.03$ \\
\hline V-line $\times 5 \%$ Potato Peel & $2.03^{c} \pm 0.02$ & $2.46^{\mathrm{d}} \pm 0.02$ & $2.21^{\mathbf{b}} \pm 0.08$ & $2.85^{c} \pm 0.06$ & $3.01^{c_{ \pm}} \pm 0.06$ & $3.51^{\mathrm{cd}} \pm 0.02$ & $2.77^{\mathrm{c}} \pm 0.10$ \\
\hline V-line $\times 10 \%$ Potato Peel & $1.96^{\mathrm{d}} \pm 0.02$ & $2.96^{\mathrm{c}} \pm 0.03$ & $2.29^{\mathbf{b}} \pm 0.07$ & $3.06^{\mathbf{b}} \pm 0.03$ & $2.80^{\mathrm{c}} \pm 0.02$ & $3.65^{c} \pm 0.09$ & $2.85^{\mathrm{c}} \pm 0.05$ \\
\hline V-line $\times 15 \%$ Potato Peel & $2.49^{c} \pm 0.07$ & $2.60^{c} \pm 0.09$ & $2.17^{\mathrm{c}} \pm 0.08$ & $3.27^{\mathbf{b}} \pm 0.09$ & $3.54^{b} \pm 0.05$ & $4.23^{\mathrm{a}} \pm 0.10$ & $3.10^{\mathrm{c}} \pm 0.08$ \\
\hline V-line $\times 20 \%$ Potato Peel & $3.34^{\mathrm{a}} \pm 0.04$ & $4.94^{\mathrm{a}} \pm 0.24$ & $3.11^{\mathrm{a}} \pm 0.55$ & $3.69^{\mathrm{a}} \pm 0.10$ & $3.95^{\mathrm{a}} \pm 0.08$ & $4.34^{\mathrm{a}} \pm 0.08$ & $3.89^{\mathrm{a}} \pm 0.03$ \\
\hline Alex-line $\times$ control & $1.87^{\mathrm{d}} \pm 0.06$ & $2.82^{c} \pm 0.12$ & $1.96^{\mathrm{d}} \pm 0.14$ & $2.73^{c_{1}} \pm 0.05$ & $2.61^{\mathrm{d}_{ \pm}} \pm 0.11$ & $3.87^{\mathbf{b}} \pm 0.13$ & $2.68^{\mathrm{d}_{ \pm}} \pm 0.09$ \\
\hline Alex-line $\times 5 \%$ Potato Peel & $2.18^{c} \pm 0.02$ & $2.39^{d_{ \pm}} \pm 0.03$ & $2.05^{\mathrm{c}} \pm 0.12$ & $2.71^{c_{ \pm}} \pm 0.03$ & $2.42^{\mathrm{d}} \pm 0.04$ & $3.35^{\mathrm{de}_{ \pm}} \pm 0.15$ & $2.57^{\mathrm{d}} \pm 0.02$ \\
\hline Alex-line $\times 10 \%$ Potato Peel & $2.01_{ \pm 0.03}^{\mathrm{d}_{ \pm}}$ & $2.70^{c} \pm 0.08$ & $1.89^{\mathrm{d}} \pm 0.08$ & $2.50^{\mathrm{d}} \pm 0.11$ & $2.41^{\mathrm{d}} \pm 0.05$ & $3.22^{\mathrm{e}} \pm 0.17$ & $2.50^{\mathrm{d}} \pm 0.04$ \\
\hline Alex-line $\times 15 \%$ Potato Peel & $1.93^{\mathrm{d}_{ \pm}} 0.03$ & $2.82^{c_{ \pm}} \pm 0.06$ & $2.32^{\mathbf{b}} \pm 0.07$ & $3.05^{\mathrm{b}} \pm 0.17$ & $3.25^{\mathrm{b}} \pm 0.04$ & $4.32^{\mathrm{a}} \pm 0.12$ & $3.00^{\mathrm{c}} \pm 0.01$ \\
\hline $\begin{array}{l}\text { Alex-line } \times 20 \% \text { Potato Peel } \\
\text { Significance }\end{array}$ & $\begin{array}{c}2.92^{\mathbf{b}} \pm 0.02 \\
* * *\end{array}$ & $\begin{array}{c}4.39^{\mathbf{b}} \pm 0.09 \\
* * *\end{array}$ & $\begin{array}{l}2.28^{\mathbf{b}} \pm 0.12 \\
* * * *\end{array}$ & $\begin{array}{c}3.31^{\mathbf{b}} \pm 0.07 \\
* * *\end{array}$ & $\begin{array}{c}3.27^{\mathbf{b}} \pm 0.04 \\
* * *\end{array}$ & $\begin{array}{c}4.17^{\mathrm{a}} \pm 0.18 \\
* * *\end{array}$ & $\begin{array}{c}3.37^{\mathbf{b}} \pm 0.06 \\
* * *\end{array}$ \\
\hline
\end{tabular}

$* * *=$ Significant at $\mathrm{p} \leq 0.001$

Different letters (a, b, c, d, e) in the same column indicate significant differences $\mathrm{p} \leq 0.05$ 
Table (11): Economic efficiency of growing rabbits lines as feeding dietary Potato Peel meal containing diets during experimental growth periods.

\begin{tabular}{|c|c|c|c|c|c|c|c|c|c|c|}
\hline \multirow{3}{*}{ Items } & \multicolumn{10}{|c|}{ Dietary Potato Peel meal, \% } \\
\hline & \multicolumn{5}{|c|}{ V-Line } & \multicolumn{5}{|c|}{ Alex. Line } \\
\hline & 0.0 & 5 & 10 & 15 & 20 & $\mathbf{0 . 0}$ & 5 & 10 & 15 & 20 \\
\hline Average feed intake/rabbit (kg) & 3.506 & 3.595 & 3.509 & 3.536 & 3.266 & 3.325 & 3.305 & 3.331 & 3.358 & 3.209 \\
\hline Price/kg diet (L.E) & 2.594 & 2.550 & 2.506 & 2.462 & 2.418 & 2.594 & 2.550 & 2.506 & 2.462 & 2.418 \\
\hline Total feed cost/ rabbit (L.E) ${ }^{1}$ & 9.095 & 9.167 & 8.794 & 8.706 & 7.897 & 8.625 & 8.428 & 8.347 & 8.267 & 7.759 \\
\hline Average body weight gain (kg) & 1.227 & 1.296 & 1.230 & 1.137 & 0.839 & 1.237 & 1.282 & 1.329 & 1.120 & 0.951 \\
\hline Price/kg body weight (L.E) & 22 & 22 & 22 & 22 & 22 & 22 & 22 & 22 & 22 & 22 \\
\hline Selling price $(\mathrm{L} . \mathrm{E})^{2}$ & 26.99 & 28.51 & 27.06 & 25.01 & 18.45 & 27.214 & 28.204 & 29.238 & 24.640 & 20.920 \\
\hline Net revenue (L.E) ${ }^{3}$ & 17.895 & 19.343 & 18.266 & 16.304 & 10.553 & 18.589 & 19.776 & 20.891 & 16.373 & 13.163 \\
\hline Economical efficiency $^{4}$ & 1.968 & 2.110 & 2.077 & 1.873 & 1.336 & 2.155 & 2.346 & 2.503 & 1.981 & 1.696 \\
\hline Relative economical efficiency ${ }^{5}$ & 100 & 107.22 & 105.54 & 95.17 & 67.89 & 100 & 108.88 & 116.14 & 91.90 & 78.72 \\
\hline
\end{tabular}

1- Feed cost $=$ feed intake $\times$ price of $\mathrm{kg}$ of diet.

2- Selling price $=$ body weight gain $\times 22 \mathrm{~L} . \mathrm{E} / \mathrm{kg}$.

3- Net revenue $=$ Selling price - feed cost.

4- Economic efficiency $=$ Net revenue $/$ feed cost.

5- Relative economic efficiency $=(\mathrm{EE} / \mathrm{EE}$ of control $) \times 100$. 


\section{REFFRENCES}

A. E. S. I., Agriculture Economic and Statistics institute, 2008. Agric., Economics, Pub. By Agric. Res. Center, Egypt.

Aboh A. B.; Zoffoun, G. A.; Djenontin, A. J. P.; Babatound; and Mensah, S. G., 2013. Effect of graded levels of dry pineapple peel o digestibility and growth performance of rabbit. J. Appl. Biosci.

Adeyemi, O. A; Ajado, A. O.; Okubanjo, A. O.; and Eniolorunda, O. O., 2010. Response of growing rabbits to graded levels of fermented and unfermented pineapple peel. ejeafche, 20: 898-909.

Al-Shanti, H. A., 2003. Effect of feeding olive cake on growth performance, nutrients digestibility, carcass traits and blood constituents of growing rabbits. Egypt. J. Rabbit Sci., 13(2): $103-116$.

Amber, K. H.; Isshak, N. S.; and ELNiamat, M.; and EL-Abd, M., 2002. Effect of dietary fiber source on performance of growing New Zeland White rabbits. Egypt. J. Poult. Sci., 22: 127-146.

A. O. A. C., Official methods of analysis Association of Official Analytical Chemists, 2000. Washington, DC, USA.

Battaa, A. M. El-Neney; Nasra, B. Awaden; Nehad, A. Ramadan; and Rizk A. M., 2013. The impact of using different levels of miswak on reproductive performance and immunity system of Dokki 4 laying hens. Egypt. Poult. Sci., 33: 865-888.

Cheeke, P. R., 1986. Potential of rabbit production in tropical and subtropical agricultural system. J. Anim. Sci., 63: $1581-1586$.

De Blas, J. C.; and Mateos, G. G.; 1998. Feed formulation. In: De Blas, J. C. and Wiseman, J. (Eds.), The nutrition of the rabbit. CABI Pub I., Wallingford, UK. Chapter 13: 241253.

Duncan, D. B., 1955. Multiple range and Multiple F-test Biometrics 11:1-42.

Eka, O. U., 1977. Studies on levels of oxalic acid and phytic acid in traditional foods of northern Nigerians. West Afri. J. Biol. Appl. Chem., 20: 26-30.

Elazab, M. A.; Zahran, S. M.; Ahmed, M. H.; and Elkomi, A. E.; 2011. Productive Performance of growing rabbits fed diet containing different levels of tomato pomace. Benha Vet. Med. J., 22(2): 44-55.

El-Boushy, A. R. Y.; and Van der Poel, A. F. B., 1994. Poultry feed from waste processing and use. Chpman and hall (Ed).

El-Raffa, A. M., 2007. Formation of a rabbit synthetic line (Alexandria line) and primary analysis of its productive and reproductive performance. Egypt. Poult. Sci., 27: 321-334.

FAO, 2006. World agriculture: towards 2030/2050 - Interim report. Rome. (http://www.fao.org/fileadmin/user_up load/esag/docs/Interim_report_AT205 0web.pdf).

Fapohunda, J. B.; Iji, O. T.; Makanjuola, B. A.; Omole, A. J., 2008. Effect of different levels of dry pineapple waste in the diet of growing rabbits.Proc. 33rd Annual Conf. Nig. Soc. Anim. Prod, pp. 195- 198.

Genedy, G. K. Salwa; Radwan, M. S. M; Zeid, A. E.; and Zanaty, G. A. 2000. Feeding rabbits dried watermelon by-product (Citrullus Lanatus Var.) as a replacing feed for clover hay. 8th Agricultural Development Research Conference, November, 20-22, Fac. Agric., Ain Shams Univ., Egypt. 
Hamendra, S. P.; Yamini, D.; and Anand Kar, 2010. Fruit and vegetable peels: Paving the way towards the development of new generation therapeutics. Drug Discov. Ther., 4(5):314-325.

Ismail, F. S. A., 2004. Wheat screening by-product in growing rabbit diets. Egypt. Poult. Sci., 14:65-79.

Khamis, M., 2014. Impact of garlic (Allium Sativum) on growth traits for two lines of rabbits under hot climates condition .M. Sc. Thesis, Fac. Agri., Alex University, Egypt.

NRC, Nutrition Requirements of Rabbits. National Research Council, 1994. 2nd Ed. Nat. Acad. Sci., Washington, DC, USA.

Offor, C. E.; Nweke, F. N.; Okaka, A. N. C.; Igwenyi, I. O.; and Onwe, V. N., 2011. Analysis of the ant nutrients levels in staple food crops in three different local government areas of ebony state, Nigeria. J. Food Sci. Tech., 5(1):26-30.

Omer, H. A. A.; Ali, F. A. F.; and Ibrahim, Sh. A. M., 2011. Strawberry By-Products as a Partial Replacement of Clover Hay in Rabbit Diets. American-Eurasian J. Agric. Environ. Sci., 11(6): 815-823.

Osagie, A. U., 1998. Anti-nutritional factors. Nutritional Quality of Plant Food, pp: 221-224.

Osagie, A. U.; and Eka, O. U., 1988. Antinutritional factors (Oxalate, Saponins, trypsin inhibitor and Alkaloid). Nutritional Quality of Plant foods. post harvest unit. University of Benin. Benin City. Nigeria, pp. 233235.

Parmar, H. S.; and Kar, A., 2009. Comparative analysis of free radical scavenging potential of several fruit peel extracts by in vitro methods. Drug Disc. Ther., 3:49-5.

Rodriguez De Sotillo, D.; Hadley, M.; Holm, E. T., 1994. Phenolics in aqueous potato peel extract: stressed rabbits. In: Proc. of the 4th International Conference on Rabbit Production in Hot Climate, Sharm ElSheikh, Egypt, 24-27 February, pp. 325- 333.

Safwat, A. M., 2010. Effect of using corn-cobs meal and dried offal of field bean in growing rabbit diets on growth performance, digestibility coefficient, carcass traits and economic efficiency. M.Sc. Thesis, Fac. Agric. Alexandria Univ., Egypt.

Sarhan, M. A., 2005. Utilization of agroindustrial by-products of pea (Pisum Sativum) in growing rabbit diets. Egypt. J. rabbit Sci., 15(2): 157 - 172.

SAS, 2003. SAS/STAT Users Guide: Version 8 for Windows. SAS Istitute Inc., Cary, NC., USA.

Schneider, B. H.; and flatt, W. P., 1975. The evaluation of feed through digestibility expermints. University of Georgia press Athens, Georgia, USA. P. 423 and terminal cross rabbits. J. App. Rabbit Res., 4:66.

Shamma, T., A.; Abdel-Azeem, F. Abdel-Azeem; and Said, M. Ismail, 2014. The effect of Incorporating treated jojoba meal in broiler diets on productive and physiological performance . Egypt. J. Poult. Sci. (34) (1): 305-331.

Singh, N.; Kamath, V.; and Rajini, P. S., 2005. Attenuation of hyperglycemia and associated biochemical parameters in STZinduced diabetic rats by dietary supplementation of potato peel powder. Clin. Chim. Acta., 353(1-2): 165-175.

Singh, $\quad$ N.; Kamath, V.; Narasimhamurthy, K., Rajini, P. S., 2008. Protective effect of potato peel extract against carbon tetrachlorideinduced liver injury in rats. Environ. Toxicol. Pharmacol., 26(2): 241-246. 
Smith, T. J.; and huxsoll, C. C., 1987. Peeling potato for processing (Eds W.F. Talburt and O. smith) AVI-Van Nostrand rein hold Company, New

Soliman, A.Z.; Selim, A.D. and A.M. Abdel-Khalek (2005). Effect of dietary crude fiber levels and supplemental lipid sources on performance of heat stressed rabbits. In: Proc. of the $4^{\text {th }}$ International Conference on Rabbit Production in Hot Climate, Sharm El-Sheikh, Egypt, 24-27 February, pp. 325- 333.

\section{الملخص العربي}

\section{الاستفادة من قثور البطاطس كمصدر للألياف في تغذية خطين من الأرانب النامية}

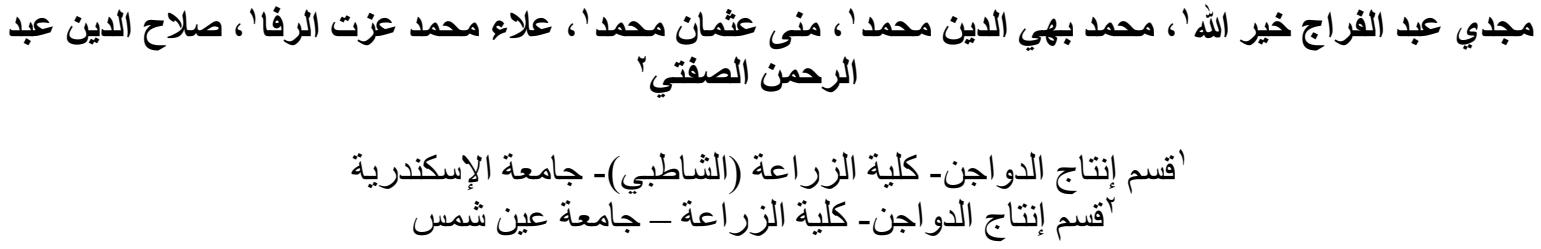

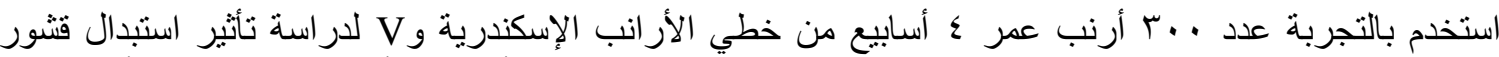

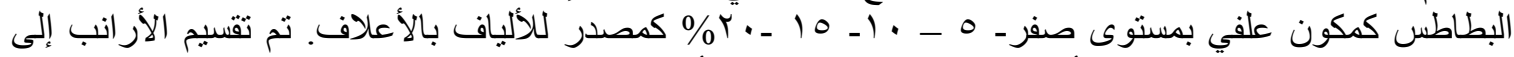

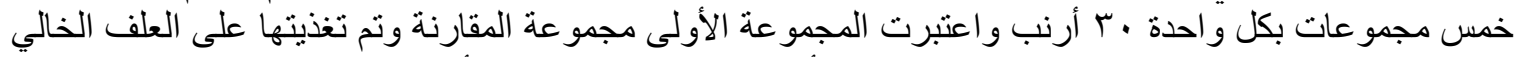

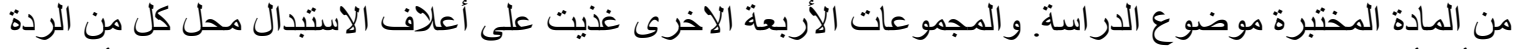

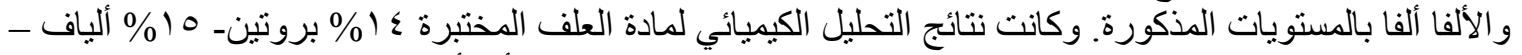

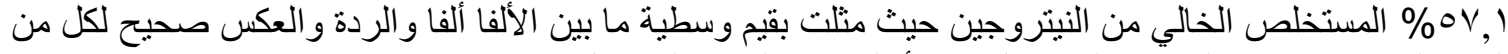

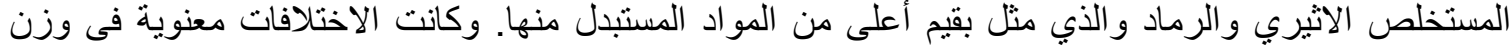

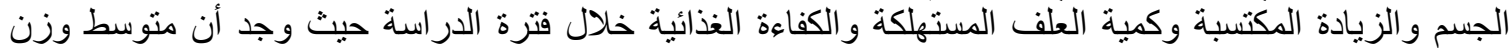

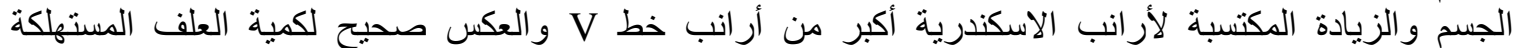

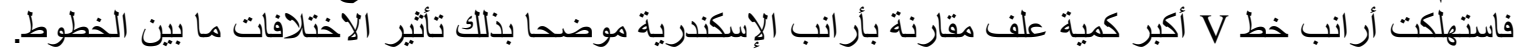

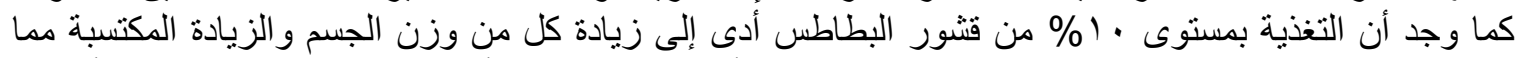

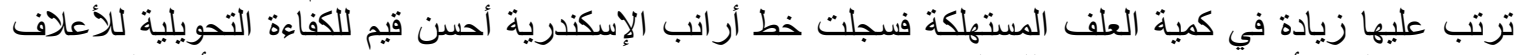

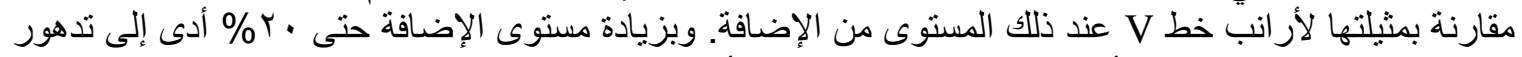

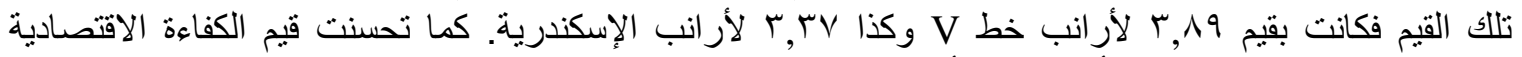

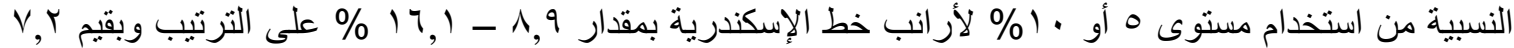

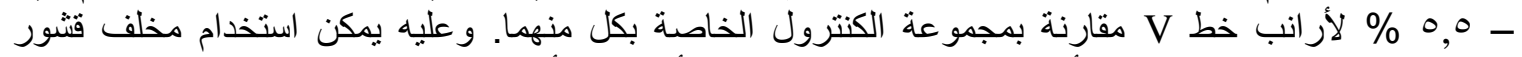

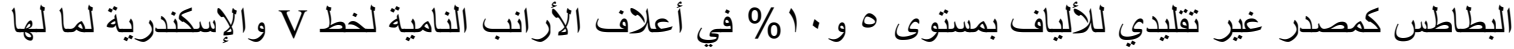
من تأثيرجيد كمصدر الألياف في تكوين أعلاف الأرانب. 